Sein allmählicher Rückzug aus der praktischen Politik, auch in seiner Landespartei, ging einher mit einer intensiveren politikwissenschaftlichen Beschäftigung: 1971 wurde Majonica an der Universität München mit einer Arbeit über die Beziehungen zwischen der Bundesrepublik Deutschland und der Volksrepublik China zwischen 1949 und 1966 promoviert. Kurz vor dem Ende seiner politischen Karriere kamen dabei seine lebenslange Beschäftigung mit der Geschichte und Kultur Asiens zum Ausdruck, aber auch seine Stärke in der theoretischen Analyse und sein überwiegend wissenschaftlich-historisches Herangehen, das in seinem außenpolitischen Denken überwog.

Nicht zuletzt die detaillierten Anmerkungen und die ausführliche Einleitung der Bearbeiter machen die vorliegende Tagebuchedition zu einem gut und mit Gewinn lesbaren Stück allgemeiner Geschichte der Außenpolitik Deutschlands und besonders der ersten beiden Jahrzehnte der Bundesrepublik.

Joachim Rott

\title{
Politische Repräsentation: Krise, Wandel, Reform?
}

Linden, Markus und Winfried Thaa (Hrsg.): Krise und Reform politischer Repräsentation, Nomos Verlagsgesellschaft, Baden-Baden 2011, €29,-.

Alonso, Sonja, John Keane und Wolfgang Merkel (Hrsg.): The Future of Representative Democracy, Cambridge University Press, Cambridge 2011, € 27,40.

Je stärker sich Gesellschaften funktional differenzieren und dabei nationalstaatliche Grenzen relativieren, desto größer werden die normativen und praktischen Herausforderungen, die mit dem zum Teil krisenhaft wahrgenommenen Wandel repräsentativer Demokratie einhergehen. In den letzten Jahren haben sich zwei Tagungsprojekte an der Universität Trier und am Wissenschaftszentrum Berlin für Sozialforschung (WZB) diesen theoretischen wie empirischen Herausforderungen gestellt und sich mit dem Status Quo sowie den Wandlungstendenzen demokratischer Repräsentation befasst. Aus diesen Auseinandersetzungen sind zwei Sammelbände hervorgegangen, die zusammengenommen einen breiten Einblick in die aktuelle Repräsentationsforschung geben. Beiden Publikationen liegt ein ähnliches Repräsentationsverständnis zugrunde: Sie wird als eine erhaltenswerte Form der Organisation demokratischer Herrschaft verstanden, die historischen Wandlungsprozessen unterworfen ist. Vor allem die Pluralisierung der Repräsentanten und der Repräsentationsbeziehungen ist ein alle Beiträge durchziehendes Thema.

Der von Markus Linden und Winfried Thaa herausgegebene Sammelband geht auf eine Tagung des Teilprojekts C 7 „Formen und Funktionsweisen politischer Repräsentation von Fremden und Armen in der Bundesrepublik Deutschland“ des SFB 600 „Fremdheit und Armut“ 2010 in Trier zurück und fragt nach Krisendiagnosen und Reformmöglichkeiten politischer Repräsentation. Die Herausgeber führen in ihrer Einleitung fünf typische Argumentationsweisen an, mit denen die Krise politischer Repräsentation beschrieben wird: Es gäbe (1) eine systematische Benachteiligung allgemeiner Interessen beziehungsweise Anliegen, und zwar (2) sowohl bei der Vermittlung als auch Durchsetzung; (3) eine zunehmenden Privatisierung und Entfremdung zwischen Staat und Bürger, (4) die zu einer Legi- 
timitätskrise führen. (5) Die daraus resultierenden Paradoxien seien Indiz für eine Steuerungskrise moderner Demokratien (S. 13 ff.). Für Reformperspektiven sehen sie in ihrem Schlusswort vier Ansatzpunkte: (1) Konfliktive Repräsentation soll zu einer stärkeren Politisierung beitragen; (2) mithilfe eines Bürger-Vetos könnte Eliten-Konsensus begegnet werden; (3) die Zivilgesellschaft und ihre Kooperation mit der Politik sollte gefördert sowie (4) die politische Verantwortlichkeit durch mehr Öffentlichkeit gestärkt werden (S. 310 ff.).

Die erste Krisendiagnose des Bandes hat Ingolf Blühdorn beigetragen. Er identifiziert ein „postdemokratisches Doppeldilemma“ (S. 45), dessen Verarbeitung zu einer „simulativen Demokratie“ (S. 69) führe. Repräsentation sei hierbei kein „authentische[s] Abbilden“, sondern „diskursive[s] zur Vorstellung bringen“ (ebenda), an dem sowohl Bürger als auch politische Eliten mitwirkten. Dass Repräsentation und Diskurs „komplementäre Operationsmodi demokratischer Politik“ (S. 91) sind, zeigt Ulrich Sarcinelli. Er verteidigt das Präsentative der Repräsentation als Grundlage einer konfliktiven Demokratie, in der über unterschiedliche Repräsentationsansprüche friedlich gestritten werden kann. Insofern sieht er eine Krise politischer Repräsentation als „demokratische Normalität“ (S. 92), die sich auf parteiisch wahrgenommene Leistungen der Akteure, nicht aber auf das demokratische System und dessen Normen und Institutionen bezieht. In Deutschland zählen die Volksparteien zu den wichtigsten Akteuren mit Repräsentationsansprüchen. Deren Bedeutungsverlust und Maßnahmen, diesem zu begegnen, analysiert Uwe Jun. Obwohl ihre „Repräsentationsmacht als Mitgliederorganisation gesunken und ihre Repräsentationschancen bei den Wählern [...] gebrochen" (S. 119) sind, könnten sie weiterhin erfolgreich aus Wahlen hervorgehen, wenn sie es schaffen, dem Wähler glaubwürdig personell und thematisch ansprechende Zukunftsperspektiven sowie neue Partizipationschancen zu präsentieren. Hieran knüpft Tha in seiner Untersuchung der Frage an, ob die „derzeitige Krise politischer Repräsentation als Resultat vorpolitischer gesellschaftlicher Veränderungen adäquat [zu] verstehen“ (S. 126) sei. Zu seinem Fazit kommt er in kritischer Auseinandersetzung mit der von Gero Neugebauer und Michael Vester vertretenen „Enttäuschungsthese“ (S. 134)1: Das Parteiensystem schafft es nicht mehr, "problematische gesellschaftliche Entwicklungen in sinnhafte politische Konfliktlinien zu transformieren“ (S. 144). In der Folge geraten auch Parlamente, deren Input- und Output-Seite kritisiert wird, unter Druck. Linden beschäftigt sich mit den identitären Repräsentations- und Parlamentsverständnissen der Krisendiagnosen von Carl Schmitt, Jürgen Habermas und Colin Crouch, die in unterschiedlichen Gewichtungen ein Hadern mit der Pluralität moderner Gesellschaften aufzeigen. Während es bei Schmitt und Habermas zu einer „monistische[n] Überhöhung “ der politischen Prinzipien „Öffentlichkeit“ und „Diskussion“ durch die Betonung der „apolitischen Merkmale (...) ,Notwendigkeit’ und ,Wahrheit'“ (S. 166) komme, könne gerade ihre konfliktive Interpretation „wesentlich mehr zur aktuellen Debatte beitragen als die Idee vom Postparlamentarismus oder die effektheischende Redeweise von der Postdemokratie“ (ebenda), wie sie beispielweise von Crouch lanciert wird.

1 Vgl. Gero Neugebauer, Politische Milieus in Deutschland. Die Studie der Friedrich-Ebert-Stiftung, Bonn 2007; Michael Vester, Soziale Milieus und Gesellschaftspolitik, in: APuZ, 56. Jg. (2006), H. 44/45, S. 10 - 17. Nach der Enttäuschungsthese schaffen es Parteien nicht mehr, die Interessen und Ansprüche sowohl der politischen als auch sozialen Milieus ausreichend zu repräsentieren. 
Die Reformperspektiven reichen von der stärkeren Nutzung symbolischer Repräsentation zur Akzeptanzverbesserung politischer Steuerung (Gerhard Göhler) bis zur Revitalisierung und Erweiterung der Demokratie in der Wirtschaft (Joachim Beerhorst). Frank Decker möchte der deutschen Repräsentationskrise durch eine Reform der politischen Systeme der Länder hin zum Präsidentialismus begegnen, da dieser Regierungstypus besser mit plebiszitären Instrumenten vereinbar sei. Jedoch profitieren bisher nicht alle von mehr direkter Demokratie, wie Brigitte Geißel in ihrer Untersuchung der Effekte lokaler partizipativer Innovationen für die deskriptive Repräsentation von und Responsivität gegenüber Fremden und Armen zeigen kann. Christiane Bausch beschäftigt sich mit den Inklusions- uns Exklusionsmechanismen deskriptiver Repräsentation am Beispiel von Ausländer- und Integrations(bei)räten. Obwohl diese die Berücksichtigung spezifischer Anliegen von ausländischen Mitbürgern und Migranten ermöglichten und ihre Existenz die Agenda von Parteien erweitere, seien vor allem kleine Gruppen und Migrantinnen nicht ausreichend repräsentiert. Zwar haben Quoten, so Annette Knaut, die deskriptive Repräsentation von Frauen im Bundestag verbessert, doch seien sie keineswegs ein Garant für die substanzielle Repräsentation frauenspezifischer Anliegen. Grund hierfür seien durch Männer geprägte und gepflegte informelle Institutionen, die bisher durch die weiblichen Abgeordneten nicht verändert worden seien beziehungsweise geändert werden konnten.

Der von Sonja Alonso, John Keane und Wolfgang Merkel herausgegebene Sammelband ist in seiner Anlage breiter und grundsätzlicher als der vor allem auf Deutschland blickende von Linden und Thaa. In ihrem ideengeschichtlich orientierten Beitrag über die repräsentative Demokratie und ihre Kritiker kommt Nadia Urbinati zu dem Schluss, dass „elections 'make“ representatives but they do not 'make‘ representation" (S. 45), und plädiert für eine stärkere Beachtung informeller Partizipation, die sich im politischen Prozess zwischen Repräsentanten und Repräsentierten durch einen kontinuierlichen Diskussionsprozess entfaltet. Eine solche prozessuale Konzeption politischer Repräsentation legt auch Michael Saward vor, der in seinen Reflexionen über das klassische Verständnis politischer Repräsentation hinausgeht. Demokratische Repräsentation sei keineswegs nur an staatliche Institutionen und Praktiken gebunden, sondern müsse von einem breiteren Blickwinkel aus betrachtet werden, um deren Grenzen neu zu bestimmen: Neben den staatlichen Repräsentanten gebe es auch andere Akteure mit Repräsentationsansprüchen, die konzeptionell zu berücksichtigen seien, sowie mehrere Ausprägungen politischer Repräsentation: permanent, temporär, territorial, nichtterritorial. Solche Akteure mit Repräsentationsansprüchen und ihre Durchsetzungschancen können sehr unterschiedlich sein, wie die dann folgenden Beiträge zeigen: Drude Dahlerup über Frauen in der repräsentativen Demokratie, Sonja Alonso über den Zusammenhang zwischen Minderheitennationalismus („minority nationalism“) und Repräsentation am Beispiel Spaniens, Robyn Eckersly über die Möglichkeiten, die Natur zu repräsentieren, John Keane über die Entwicklung der repräsentativen Demokratie zu einer „Monitory Democracy“, die gekennzeichnet ist durch ",a variety of 'post-parliamentary' politics defined by the rapid growth of many different kinds of extra-parliamentary, power-scrutinising mechanisms" (S. 212), sowie Michael Zürn und Gregor Walter-Drop über Repräsentation jenseits des Nationalstaats.

Neben diesen detailreichen theoretisch-konzeptionellen Beiträgen finden sich empirisch ausgerichtete Aufsätze: Klaus von Beyme beschäftigt sich mit drei kritischen Interpretationsweisen und Entwicklungstendenzen gegenwärtiger Demokratien: der wachsenden Zahl defekter Demokratien, der Zunahme politischen Populismus' und der Forderung nach mehr 
direkter Demokratie. Auch Bernhard Weßels befasst sich vergleichend mit der Qualität politischer Repräsentation heutiger Demokratien. Obwohl ein geringes Vertrauen in Parteien und Politiker damit erklärbar und durchaus relativierbar sei, dass Bürger ihren favorisierten Parteien und Politikern mehr vertrauten als anderen, müsse zu denken geben, dass Bürger den Wahlen immer weniger zutrauen, ihre Funktion zu erfüllen, politische Repräsentation zu gewährleisten. Dies gilt umso mehr, als demokratische Legitimität erheblich davon abhänge, dass Wahlen als bedeutsam und einen Unterschied machend wahrgenommen werden.

Mit den Problemen und der Zukunftsfähigkeit des in modernen Demokratien zentralen Repräsentationsorgans Parlament beschäftigt sich David Beetham. Repräsentative Versammlungen mit Gesetzgebungs- und Budgetrechten seien auch künftig unverzichtbar, so dass deren öffentlicher Ansehensverlust sehr ernst sei: „Only a reinvention of parliaments that goes further than anything envisaged will meet the demands of the situation." (S. 138) Parlamente hätten jedoch hierfür gute Voraussetzungen, da sie sich stets als reformfähig erwiesen haben, wichtige Institutionen der Öffentlichkeit und deren Herstellung seien und die inzwischen deutlich bessere Bildung der Bevölkerung neue Kontaktmöglichkeiten eröffnet (S. 139 f.).

Für Phillipe Schmitter sind all dies Indizien für mehrere gleichzeitig stattfindende ,'postDahlian“ revolutions“ (S. 193) der Demokratie in Europa, wobei gegenwärtig die „klassischen" demokratischen Akteure Bürger, Interessenorganisationen und politischen Repräsentanten zugleich von den Umwälzungen derart betroffen sind, dass die Veränderungen als krisenhaft beschrieben werden können. Merkmale seien (1) die Ersetzung von Individuen durch Organisationen als "effective citizens of democracy“ (S. 194), (2) die Professionalisierung der Politiker, (3) die Herausbildung von politisch unabhängigen Wächterinstitutionen wie Zentralbanken und Kartellbehörden, (4) die Ausbildung von Formen des Mehrebenenregierens und (5) die zunehmende Bedeutung von "good governance"Kriterien. Und während Krisen bisher stets zu demokratischen Experimenten geführt hätten, gelte heute: „The 'Great Recession' that they [heutige Demokratien] are currently suffering seems to have dampened rather than enhanced the likelihood of such experiments." (S. 209)

Beide Bände zusammengenommen können zum Teil bereits bekannte Forschungspositionen zum Thema kompakt und problembewusst wieder vor Augen führen, gegebenenfalls auch als Seminartexte dienen und Einsteigern in die Welt der Repräsentationsforschung durch die Vielfältigkeit der Ansatzmöglichkeiten Anregungen für weitere Lektüre und Forschungen sein. Leider fehlt beiden Bänden ein Aufsatz, der die empirische Repräsentationsforschung in der Tradition von Heinz Eulau, John C. Wahlke u.a., Warren E. Miller, Donald E. Stokes sowie Hanna Pitkin kritisch hinsichtlich ihres Potenzials, die heutigen Wandlungstendenzen adäquat zu erfassen, beleuchtet. Dennoch fordern sowohl die Artikel, die generelle Entwicklungen aufzeigen, als auch diejenigen, die Einzelaspekte vertiefen, zum Nachdenken und Diskutieren über die Beschaffenheit und die Zukunft eines der wichtigsten Organisationsprinzipien demokratischer Herrschaft auf. 